DOI: $10.17516 / 1998-2836-0168$

УДК 666.762.09:544.46

\title{
Effect of the Mechanical and Thermal Prehistory of Precursors on the Zinc Spinel Synthesis
}

\author{
Nadezhda F. Kosenko, \\ Natalya V. Filatova* and Viktoriia I. Rodionova \\ Ivanovo State University of Chemistry and Technology \\ Ivanovo, Russian Federation
}

Received 07.10.2019, received in revised form 05.11.2019, accepted 25.12.2019

\begin{abstract}
Thezinc spinel (gahnite) formation by a treatment of reaction mixtures of $\mathrm{Zn}$ and $\mathrm{Al}$ oxides, hydroxides, and salts was investigated. The influence of a preliminary mechanical activation by impact (planetary mill) and attrition (ball-ring mill) action as well as a microwave treatment was analyzed. The combined method consisting of a mechanical treatment of nitrates mixture in a planetary mill and the subsequent burning in a thermal kiln was considered as the most effective as it resulted in practically single product.
\end{abstract}

Keywords: zinc spinel, gahnite, solid-phase synthesis, mechanical activation, microwave treatment.

Citation: Kosenko N.F., Filatova N.V., Rodionova V.I. Effect of the mechanical and thermal prehistory of precursors on the zinc spinel synthesis, J. Sib. Fed. Univ. Chem., 2020, 13(1), 53-64. DOI: 10.17516/1998-2836-0168

(C) Siberian Federal University. All rights reserved

This work is licensed under a Creative Commons Attribution-NonCommercial 4.0 International License (CC BY-NC 4.0).

* Corresponding author E-mail address: zyanata@mail.ru 


\title{
Влияние механической
}

\section{и термической предыстории прекурсоров \\ на синтез цинковой шпинели}

\author{
Н.Ф. Косенко, Н.В. Филатова, В.И. Родионова \\ Ивановский государственный \\ химико-технологический университет \\ Российская Федерачия, Иваново
}

\begin{abstract}
Аннотация. Исследовано накопление цинковой шпинели (ганита) обработкой реакционных смесей, состоящих из оксидов, гидроксидов, солей $\mathrm{Zn}$ и Al. Проанализировано влияние предварительной микроволновой обработки и механоактивации ударным и истирающим способами в планетарной и шаро-кольцевой мельницах. Установлено, что наиболее эффективный комбинированный способ, приводящий к образованию практически монофазного продукта, состоит из механической обработки смеси нитратов в планетарной мельнице и обжига в термической печи.
\end{abstract}

Ключевые слова: шпинель, ганит, твердофазный синтез, механоактивация, микроволновая обработка.

Цитирование: Косенко, Н.Ф. Влияние механической и термической предыстории прекурсоров на синтез цинковой шпинели / Н.Ф. Косенко, Н.В. Филатова, В.И. Родионова // Журн. Сиб. федер. ун-та. Химия, 2020. 13(1). С. 53-64. DOI: $10.17516 / 1998-2836-0168$

\section{Введение}

Многие десятилетия не снижается интерес к шпинелям. Это вызвано уникальным комплексом свойств соединений, способствующим их разнообразному применению, которое непрерывно расширяется. Алюминат цинка $\mathrm{ZnAl}_{2} \mathrm{O}_{4}$ (ганит) устойчив до температуры $\sim 1950{ }^{\circ} \mathrm{C}$, полупроводник, проявляет высокую механическую прочность и термостойкость, каталитические свойства $[1,2]$. Ганит используют в керамической и электронной промышленности $[3,4]$, в каталитических процессах [5] как фотокатализатор [6], сорбент [7] и т.п. Допированный ганит обладает люминесцентными свойствами [8]. $\mathrm{ZnAl}_{2} \mathrm{O}_{4}$ - светоотражающий пигмент белого цвета, пригодный для окраски тепловых двигателей, обшивки печей, авиатехники и др. [8].

Алюминат цинка получают различными способами: керамическим синтезом [3], соосаждением [9], золь-гель методом [10], горением [11], гидротермальным способом [12], с помощью ультразвука [7], плазмы [4]. Но для промышленных целей основным остается твердофазный синтез - взаимодействие между оксидами цинка и алюминия при 1100-1600 ${ }^{\circ} \mathrm{C}$. Данный процесс является длительным и энергоемким, поэтому его пробуют сопровождать микроволновой [13] или механической обработкой [14].

$\mathrm{B}$ данной работе проанализирован керамический синтез $\mathrm{ZnAl}_{2} \mathrm{O}_{4}$, сочетаемый с микроволновой и механической обработкой различного типа. 


\section{Экспериментальная часть}

Были использованы реактивы: оксид цинка $\mathrm{ZnO}$, гидроксид цинка $\mathrm{Zn}(\mathrm{OH})_{2}$, гидроксид алюминия $\mathrm{Al}(\mathrm{OH})_{3}$, нитрат цинка $\mathrm{Zn}\left(\mathrm{NO}_{3}\right)_{2} \cdot 6 \mathrm{H}_{2} \mathrm{O}$, нитрат алюминия $\mathrm{Al}\left(\mathrm{NO}_{3}\right)_{3} \cdot 9 \mathrm{H}_{2} \mathrm{O}$, ацетат цинка $\mathrm{Zn}\left(\mathrm{CH}_{3} \mathrm{COO}\right)_{2} \cdot 2 \mathrm{H}_{2} \mathrm{O}$ квалификации «ч.д.а.», активный оксид алюминия $\mathrm{Al}_{2} \mathrm{O}_{3}$ (марка AOA-1) («х.ч.»). Исходные компоненты брали в количествах, обеспечивающих соотношение $\mathrm{ZnO}: \mathrm{Al}_{2} \mathrm{O}_{3}=1: 1$ в продукте.

Механическую обработку смесей осуществляли в планетарной и шаро-кольцевой мельницах, а СВЧ-обработку (15 мин) - в микроволновой печи марки BOSCHHMT72M420 (2.45 МГц).

Рентгенофазовый анализ выполняли на дифрактометре ДРОН-6 ( $\mathrm{Cu}_{\mathrm{K \alpha}}$-излучение). Соединения идентифицировали с помощью базы данных ASTM-JCPDS. Параметр решетки шпинели рассчитывали по дифрактометрическим данным. Размеры областей когерентного рассеяния

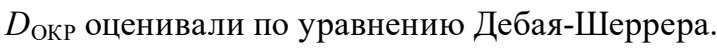

ИК-спектры образцов (таблеток с KBr) снимали на приборе BrukerFTIRIFS-88.

Удельную поверхность определяли на приборе Autosorb-1 Quantachrome по изотермам адсорбции азота с последующим расчетом по методу БЭТ.

Для изучения кинетики таблетки готовили с двухстадийным прессованием смеси (200 МПа). В качестве временной связки брали 40\%-ный раствор полиакриловой кислоты.

Образцы обжигали в печах ТИП МП 2УМ и SNOL 6.7/1300,IP20.

\section{Результаты и обсуждение}

На рис. $1 a$ приведены значения степени превращения (содержания шпинели) $\alpha$ в зависимости от времени. Данные обработали с применением различных моделей, предполагающих в качестве лимитирующей стадии объемную диффузию. Расчеты показали, что кинетика изученной реакции удовлетворительно описывается уравнением Гинстлинга-Броунштейна:

$$
1-\frac{2}{3} \alpha-(1-\alpha)^{2 / 3}=k_{\Gamma Б} \tau
$$

где $\alpha$ - степень превращения в долях единицы; $k_{\Gamma ь}-$ эффективная константа скорости, $\mathrm{c}^{-1} ; \tau-$ время, с.

Это подтверждается прямолинейностью зависимостей в координатах данного уравнения (рис. 1б) с достаточно высоким коэффициентом линейной аппроксимации на большой глубине реакции. В табл. 1 приведены эффективные константы скорости реакции для различных прекурсоров.

Гидроксиды и соли, испытывающие термолиз перед шпинелеобразованием, формируют активные оксиды, которые взаимодействуют с большей скоростью. Однако и в этих случаях скорость реакции мала. Лишь при сильном уплотнении (200 МПа) реакционных смесей взаимодействие протекает достаточно активно.

Сопоставлены дифрактограммы продукта обжига $\left(400,800,1000,1150{ }^{\circ} \mathrm{C}, 2\right.$ ч) смесей, полученных простым перемешиванием и совместным истиранием компонентов $\mathrm{ZnO}$ и $\mathrm{Al}(\mathrm{OH})_{3}$. Обжиг при $400{ }^{\circ} \mathrm{C}$ не приводит к появлению продукта. Все линии на дифрактограммах принадлежат исходным веществам. После обжига смесей при $800^{\circ} \mathrm{C}$ продукт содержит некоторое 


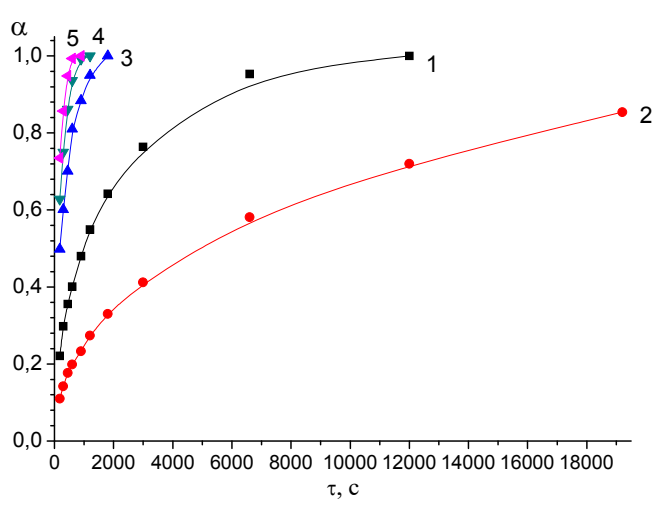

$a$

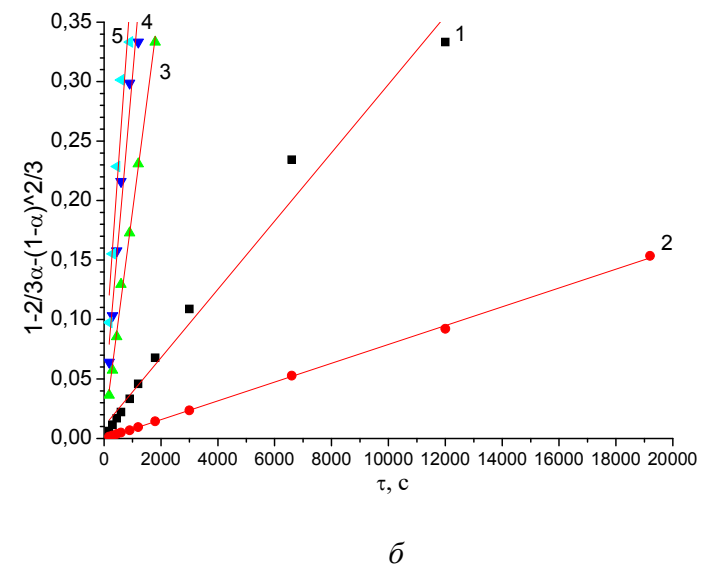

б

Рис. 1. Кинетические зависимости степени превращения $\alpha(a)$ и в координатах уравнения ГинстлингаБроунштейна (б) для реакции образования шпинели $\mathrm{ZnAl}_{2} \mathrm{O}_{4}$ из смеси: $1-\mathrm{ZnO}+\mathrm{Al}_{2} \mathrm{O}_{3}\left(1000{ }^{\circ} \mathrm{C}\right) ; 2-\mathrm{ZnO}$ $+\mathrm{Al}_{2} \mathrm{O}_{3}\left(800{ }^{\circ} \mathrm{C}\right) ; 3-\mathrm{ZnO}+\mathrm{Al}(\mathrm{OH})_{3}\left(1000^{\circ} \mathrm{C}\right) ; 4-\mathrm{Zn}(\mathrm{OH})_{2}+\mathrm{Al}(\mathrm{OH})_{3}\left(1000^{\circ} \mathrm{C}\right) ; 5-\mathrm{Zn}\left(\mathrm{CH}_{3} \mathrm{OO}\right)_{2}+\mathrm{Al}(\mathrm{OH})_{3}$ $\left(1000^{\circ} \mathrm{C}\right)$

Fig. 1. Kinetic relations of conversion degree of $\alpha(a)$ and in coordinates of Gistling-Braunstein equation ( $\sigma)$ for $\mathrm{ZnAl}_{2} \mathrm{O}_{4}$ spinel formation reaction from mixture: $1-\mathrm{ZnO}+\mathrm{Al}_{2} \mathrm{O}_{3}\left(1000{ }^{\circ} \mathrm{C}\right) ; 2-\mathrm{ZnO}+\mathrm{Al}_{2} \mathrm{O}_{3}\left(800{ }^{\circ} \mathrm{C}\right) ; 3-\mathrm{ZnO}$ $+\mathrm{Al}(\mathrm{OH})_{3}\left(1000{ }^{\circ} \mathrm{C}\right) ; 4-\mathrm{Zn}(\mathrm{OH})_{2}+\mathrm{Al}(\mathrm{OH})_{3}\left(1000{ }^{\circ} \mathrm{C}\right) ; 5-\mathrm{Zn}\left(\mathrm{CH}_{3} \mathrm{OO}\right)_{2}+\mathrm{Al}(\mathrm{OH})_{3}\left(1000{ }^{\circ} \mathrm{C}\right)$

Таблица 1. Эффективные константы скорости реакции образования $\mathrm{ZnAl}_{2} \mathrm{O}_{4}$

Table 1. Effective constants for formation reaction rate of $\mathrm{ZnAl}_{2} \mathrm{O}_{4}$

\begin{tabular}{|l|c|c|c|}
\hline \multicolumn{1}{|c|}{ Прекурсоры } & $\begin{array}{c}\text { Температура } \\
\text { керамического синтеза, }{ }^{\circ} \mathrm{C}\end{array}$ & $\begin{array}{c}\text { Эффективная константа } \\
\text { скорости } \mathrm{k}_{\text {Гь }} \cdot 10^{5}, \mathrm{c}^{-1}\end{array}$ & $\begin{array}{c}\text { Коэффициент линейной } \\
\text { аппроксимации } \mathrm{R}\end{array}$ \\
\hline $\mathrm{ZnO}+\mathrm{Al}_{2} \mathrm{O}_{3}(\mathrm{AOA})$ & 1000 & $3.4 \pm 0.5$ & 0.979 \\
\hline $\mathrm{ZnO}+\mathrm{Al}_{2} \mathrm{O}_{3}(\mathrm{AOA})$ & 800 & $0.79 \pm 0.02$ & 0.999 \\
\hline $\mathrm{ZnO}+\mathrm{Al}(\mathrm{OH})_{3}$ & 1000 & $20 \pm 1$ & 0.996 \\
\hline $\mathrm{Zn}(\mathrm{OH})_{2}+\mathrm{Al}(\mathrm{OH})_{3}$ & 1000 & $34 \pm 3$ & 0.958 \\
\hline $\mathrm{Zn}\left(\mathrm{CH}_{3} \mathrm{OO}\right)_{2}+\mathrm{Al}(\mathrm{OH})_{3}$ & 1000 & $49 \pm 7$ & 0.886 \\
\hline $\mathrm{Zn}\left(\mathrm{NO}_{3}\right)_{2}+\mathrm{Al}\left(\mathrm{NO}_{3}\right)_{3}$ & 1000 & $61 \pm 5$ & 0.912 \\
\hline
\end{tabular}

количество ганита $\left(\mathrm{ZnAl}_{2} \mathrm{O}_{4}\right)$ (рис. $\left.2 a, \sigma\right)$. Максимальный пик $\mathrm{ZnAl}_{2} \mathrm{O}_{4}$ в случае простого смешения имеет интенсивность, равную $16 \%$, а после истирания 25 \%. Для 73 \% пика ганита интенсивность составляет 12 и 19 \% соответственно. Еще в большей степени эта закономерность проявляется при повышенных температурах (рис. 2в-е). Обжиг при $1150{ }^{\circ} \mathrm{C}$ после перетирания смеси реагентов приводит к формированию почти однофазного ганита. Интенсивность максимальных рефлексов $\mathrm{ZnO}$ не превышает 18-21 \%. Свободный $\mathrm{Al}_{2} \mathrm{O}_{3}$ не обнаружен (возможно, он присутствует в рентгеноаморфной фазе).

Для понижения параметров процесса с достижением высокого выхода продукта смеси $\mathrm{Zn}(\mathrm{OH})_{2}+\mathrm{Al}(\mathrm{OH})_{3}, \mathrm{Zn}\left(\mathrm{NO}_{3}\right)_{2}+\mathrm{Al}(\mathrm{OH})_{3}, \mathrm{Zn}(\mathrm{OH})_{2}+\mathrm{Al}\left(\mathrm{NO}_{3}\right)_{3}$ и $\mathrm{Zn}\left(\mathrm{NO}_{3}\right)_{2}+\mathrm{Al}\left(\mathrm{NO}_{3}\right)_{3}$ с соотношением компонентов, рассчитанным на получение шпинели, активировали микроволновой (MBO) и механической обработкой (МО). 


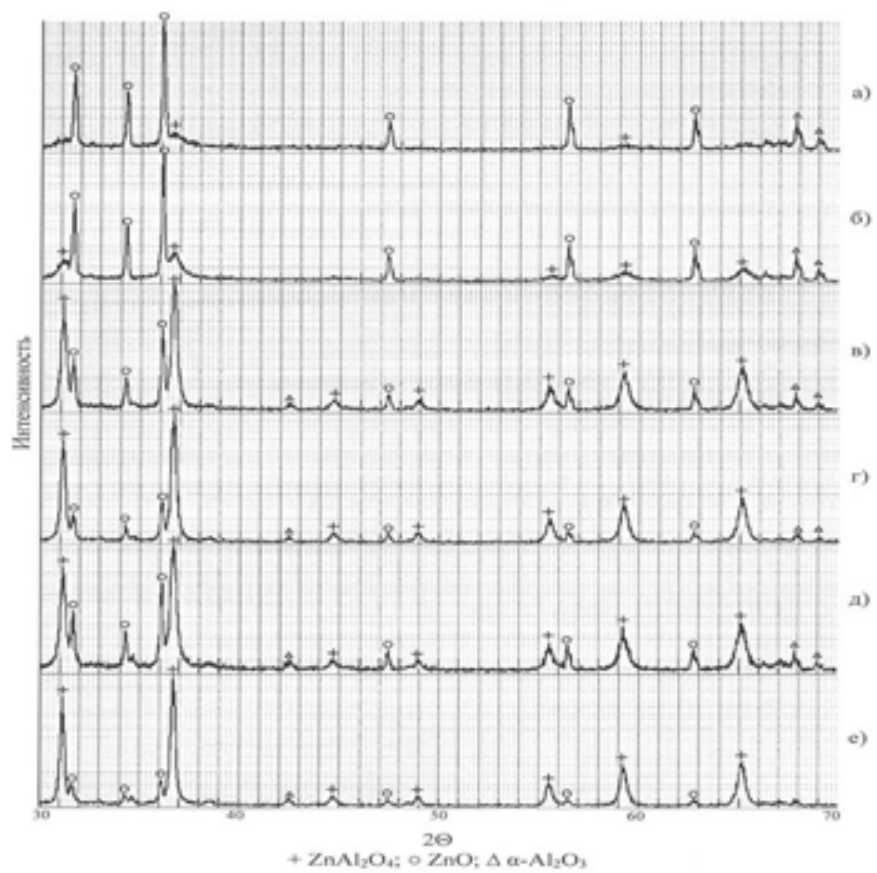

Рис. 2. Дифрактограммы продукта обжига смеси $\mathrm{ZnO}$ и $\mathrm{Al}(\mathrm{OH})_{3}$ после простого смешения $(a, 6, \partial)$ и истирающей обработки $(\sigma, 2, e)$; температура обжига, ${ }^{\circ} \mathrm{C}: a, \sigma-800 ; 6,2-1000 ; \partial, e-1150(2$ ч)

Fig. 2. Diffractograms of $\mathrm{ZnO}$ and $\mathrm{Al}(\mathrm{OH})_{3}$ mixture kilning products after simple mixing $(a, b, \partial)$ and abrasive treatment $(\sigma, 2, e)$; burning temperature, ${ }^{\circ} \mathrm{C}: a, \sigma-800 ; 6,2-1000 ; \partial, e-1150$ (2 hours)

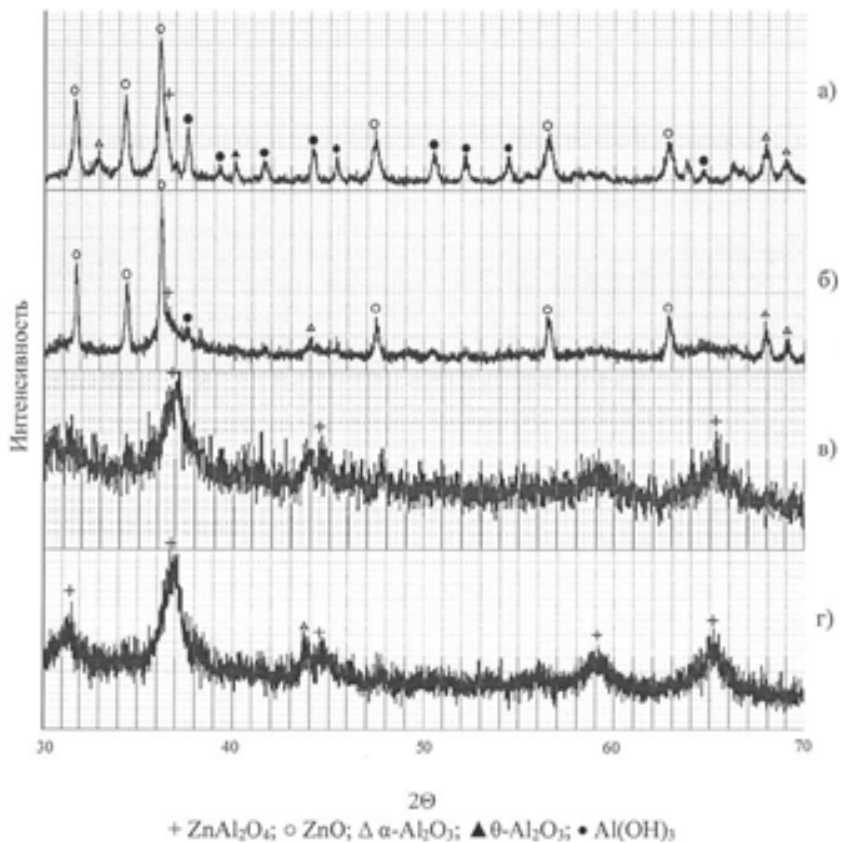

Рис. 3. Дифрактограммы реакционных смесей после микроволновой обработки (15 мин): $a-\mathrm{Zn}(\mathrm{OH})_{2}$ и $\mathrm{Al}(\mathrm{OH})_{3} ; \sigma-\mathrm{Zn}\left(\mathrm{NO}_{3}\right)_{2}$ и $\mathrm{Al}(\mathrm{OH})_{3} ;$ в $-\mathrm{Zn}(\mathrm{OH})_{2}$ и $\mathrm{Al}\left(\mathrm{NO}_{3}\right)_{3} ; 2-\mathrm{Zn}\left(\mathrm{NO}_{3}\right)_{2}$ и $\mathrm{Al}\left(\mathrm{NO}_{3}\right)_{3}$

Fig. 3. Diffractograms of reaction mixtures after microwave treatment for 15 minutes: $a-\mathrm{Zn}(\mathrm{OH})_{2}$ and $\mathrm{Al}(\mathrm{OH})_{3}$; $6-\mathrm{Zn}\left(\mathrm{NO}_{3}\right)_{2}$ and $\mathrm{Al}(\mathrm{OH})_{3} ; 6-\mathrm{Zn}(\mathrm{OH})_{2}$ and $\mathrm{Al}\left(\mathrm{NO}_{3}\right)_{3} ; 2-\mathrm{Zn}\left(\mathrm{NO}_{3}\right)_{2}$ and $\mathrm{Al}\left(\mathrm{NO}_{3}\right)_{3}$ 
Дифрактограммы продуктов после МВО представлены на рис. 3.

$\mathrm{B}$ смесях $\mathrm{Zn}(\mathrm{OH})_{2}+\mathrm{Al}(\mathrm{OH})_{3}$ и $\mathrm{Zn}(\mathrm{OH})_{2}+\mathrm{Al}\left(\mathrm{NO}_{3}\right)_{3}$ после $\mathrm{MBO}$ появляются оксидные формы: немного $\alpha-\mathrm{Al}_{2} \mathrm{O}_{3}$ и значительное количество $\mathrm{ZnO}$. В ходе $\mathrm{MBO}$ смесей $\mathrm{Zn}\left(\mathrm{NO}_{3}\right)_{2}+\mathrm{Al}(\mathrm{OH})_{3}$ и $\mathrm{Zn}\left(\mathrm{NO}_{3}\right)_{2}+\mathrm{Al}\left(\mathrm{NO}_{3}\right)_{3}$ образуется слабо закристаллизованный ганит $\mathrm{ZnAl}_{2} \mathrm{O}_{4}$. Поскольку рентгенофазовый анализ в таких случаях не всегда позволяет получить информацию о составе веществ, был выполнен ИК-спектральный анализ компонентов (рис. 4).

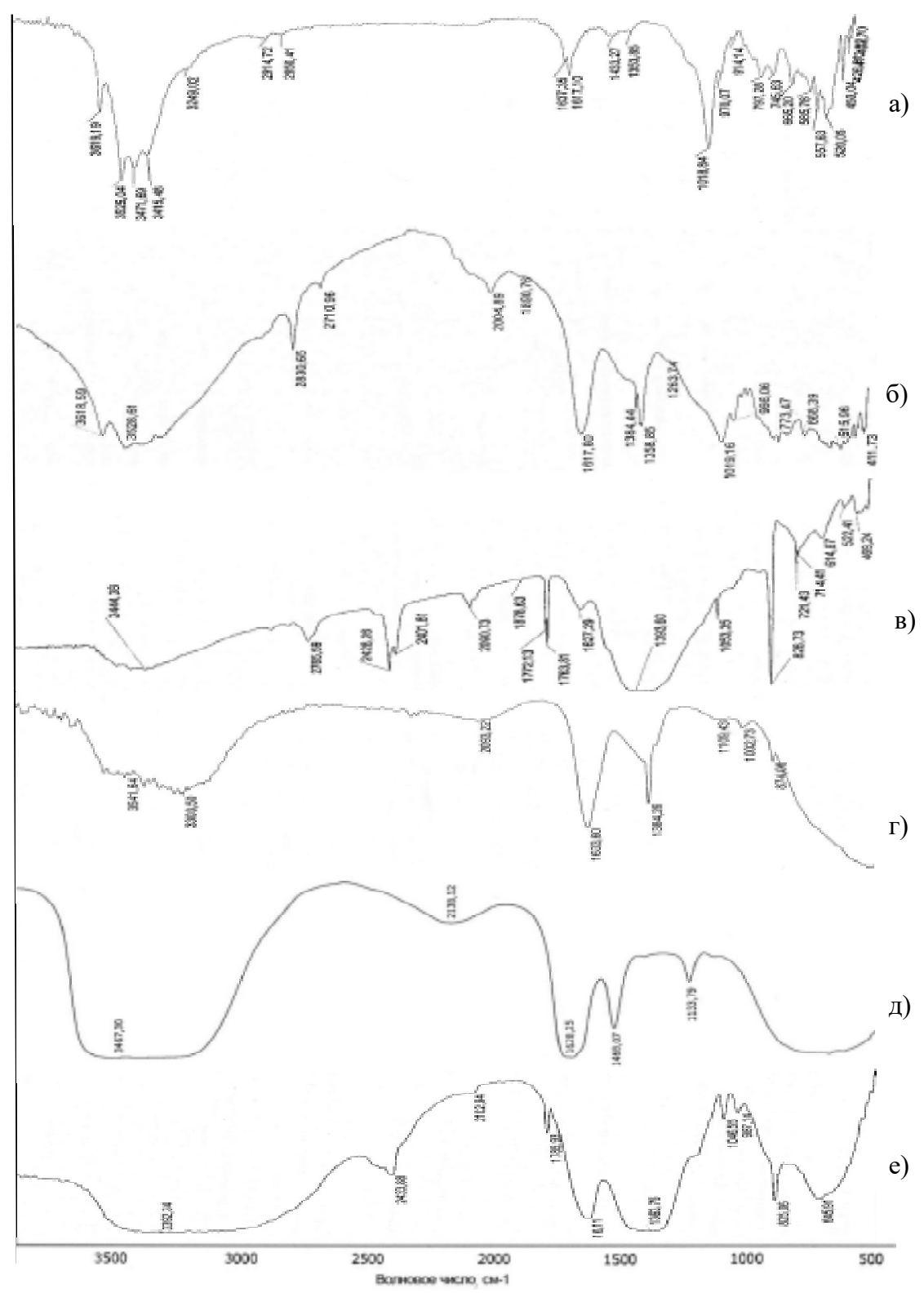

Рис. 4. ИК-спектры $\mathrm{Al}(\mathrm{OH})_{3}(a, \sigma), \mathrm{Zn}\left(\mathrm{NO}_{3}\right)_{3} \cdot 6 \mathrm{H}_{2} \mathrm{O}(6$, , $), \mathrm{Al}\left(\mathrm{NO}_{3}\right)_{3} \cdot 9 \mathrm{H}_{2} \mathrm{O}(\partial, e)$ без микроволновой обработки $(a, b, \partial)$ и после микроволновой обработки в течение 15 мин $(\sigma, 2, e)$

Fig. 4. IR spectrum of $\mathrm{Al}(\mathrm{OH})_{3}(a, \sigma), \mathrm{Zn}\left(\mathrm{NO}_{3}\right)_{3} \cdot 6 \mathrm{H}_{2} \mathrm{O}(6,2), \mathrm{Al}\left(\mathrm{NO}_{3}\right)_{3} \cdot 9 \mathrm{H}_{2} \mathrm{O}(\partial, e)$ without microwave treatment $(a, b, \partial)$ and after microwave treatment for 15 minutes $(\sigma, 2, e)$ 
Гидроксид алюминия (рис. $4 a$ ) содержит полосы поглощения, соответствующие валентному колебанию изолированных ОН-групп ( $\left.3620 \mathrm{~cm}^{-1}\right)$. Хорошо разрешенные полосы в областях 3415-3525 и 1617-1637 см-1 указывают на то, что большая часть гидроксогрупп, по-видимому, связана водородными связями. После МВО (рис. 4б) эти полосы сливаются в сравнительно широкие полосы кристаллогидратной воды, т.е. гидроксид $\mathrm{Al}$ в большей степени соответствует оксиду $\mathrm{Al}_{2} \mathrm{O}_{3} \cdot n \mathrm{H}_{2} \mathrm{O}$. Удаление части воды при этом маловероятно, т.к. оксидные формы дифрактометрически не обнаруживаются.

В исходных нитратах цинка и алюминия (рис. 46, $\partial$ ) полосы в области 3600-3300(3000) $\mathrm{cm}^{-1}$ являются сложными, состоящими как минимум из 2-3 более узких полос, что указывает на различную прочность связи молекул кристаллогидратной воды с основой. После МВО (рис. 42, e) эти полосы становятся более широкими, что может указывать на увеличение в различиях степени связывания кристаллогидратной воды. Нитрат-анионы при этом не разрушаются, что подтверждается наличием полос 1633-1616 cm c $^{-1}$ (асимметричные валентные колебания $v_{\mathrm{as}} \mathrm{ONO}_{2}$ ) и 1384-1364 см $\mathrm{cm}^{-1}\left(v_{\mathrm{s}} \mathrm{NO}_{2}\right.$-групп). Учитывая различия в форме этих полос (их расщепление и слияние), можно предположить некоторую деформацию анионов.

Поскольку именно в системах с участием нитрата алюминия после МВО появляется целевой продукт ганит, по-видимому, слабо связанная кристаллогидратная вода легко удаляется, а деформированные нитратные группы разлагаются. В результате образуется высокоактивный оксид алюминия, способствующий шпинелеобразованию. Сформированный ганит является слабозакристаллизованным (рис. $5 a$, б). Имеются также рентгеноаморфные исходные и промежуточные соединения.

В ходе МВО в уплотненных смесях $\mathrm{Zn}(\mathrm{OH})_{2}+\mathrm{Al}(\mathrm{OH})_{3}$ и $\mathrm{Zn}\left(\mathrm{NO}_{3}\right)_{2}+\mathrm{Al}(\mathrm{OH})_{3}$ шпинель не образуется (табл. 2). В обожженной массе из $\mathrm{Zn}(\mathrm{OH})_{2}+\mathrm{Al}\left(\mathrm{NO}_{3}\right)_{3}$ образуется смесь примерно равных количеств шпинели и $\mathrm{ZnO}$; кроме того, имеется много рентгеноаморфной фазы (РАФ). В смеси нитратов $\mathrm{Zn}$ и $\mathrm{Al}$ после уплотнения и $\mathrm{MBO}$ преобладает ганит, а также присутствует

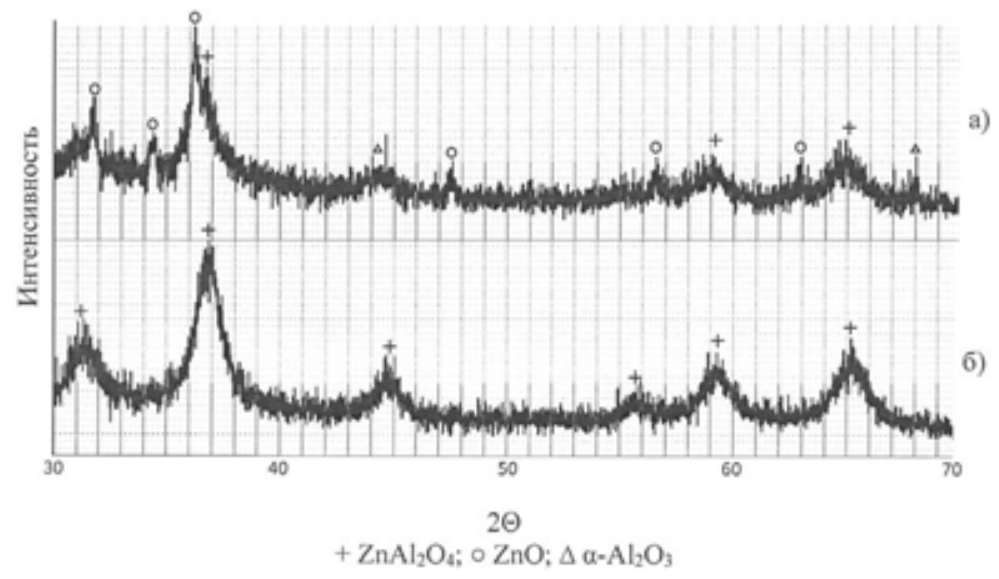

Рис. 5. Дифрактограммы спрессованных реакционных смесей после микроволновой обработки (15 мин): $a-\mathrm{Zn}(\mathrm{OH})_{2}$ и $\mathrm{Al}\left(\mathrm{NO}_{3}\right)_{3} ; \sigma-\mathrm{Zn}\left(\mathrm{NO}_{3}\right)_{2}$ и $\mathrm{Al}\left(\mathrm{NO}_{3}\right)_{3}$

Fig. 5. Diffractograms ofpressed reaction mixtures after microwave treatment for 15 minutes: $a-\mathrm{Zn}(\mathrm{OH})_{2}$ and $\mathrm{Al}\left(\mathrm{NO}_{3}\right)_{3} ; \sigma-\mathrm{Zn}\left(\mathrm{NO}_{3}\right)_{2}$ and $\mathrm{Al}\left(\mathrm{NO}_{3}\right)_{3}$ 
Таблица 2. Сравнительный анализ фазового состава продуктов термообработки смесей различного состава

Table 2. Comparative analysis of phase composition different mixtures products thermal treatment

\begin{tabular}{|c|c|c|c|}
\hline \multirow{2}{*}{$\begin{array}{c}\text { Состав } \\
\text { исходной смеси }\end{array}$} & \multicolumn{3}{|c|}{ Фазы с максимальной величиной рефлекса после обработки } \\
\hline & в СВЧ-печи & $\begin{array}{c}\text { в СВЧ-печи } \\
\text { (прессов.) }\end{array}$ & $\begin{array}{c}\text { в СВЧ- и } \\
\text { термической печи }\end{array}$ \\
\hline $\mathrm{Zn}(\mathrm{OH})_{2}+\mathrm{Al}(\mathrm{OH})_{3}$ & $\begin{array}{c}\mathrm{Al}(\mathrm{OH})_{3}(100 \%) \\
\mathrm{ZnO}(69 \%)\end{array}$ & $\begin{array}{c}\mathrm{Al}(\mathrm{OH})_{3}(100 \%) \\
\mathrm{ZnO}(42 \%)\end{array}$ & $\begin{array}{c}\mathrm{ZnO}(100 \%) \\
\mathrm{ZnAl}_{2} \mathrm{O}_{4}(80 \%)\end{array}$ \\
\hline $\mathrm{Zn}(\mathrm{OH})_{2}+\mathrm{Al}\left(\mathrm{NO}_{3}\right)_{3}$ & $\begin{array}{c}\mathrm{ZnAl}_{2} \mathrm{O}_{4}(100 \%), \\
\text { переходные формы } \mathrm{Al}_{2} \mathrm{O}_{3} \\
(70 \%), \mathrm{ZnO}(57 \%), \text { очень } \\
\text { много РАФ }\end{array}$ & $\begin{array}{c}\mathrm{ZnAl}_{2} \mathrm{O}_{4}, \mathrm{ZnO} \text { (примерно } \\
\text { равное количество), } \\
\text { много РАФ }\end{array}$ & $\begin{array}{c}\mathrm{ZnAl}_{2} \mathrm{O}_{4}(100 \%) \\
\mathrm{ZnO}(32 \%)\end{array}$ \\
\hline $\mathrm{Zn}\left(\mathrm{NO}_{3}\right)_{2}+\mathrm{Al}(\mathrm{OH})_{3}$ & $\begin{array}{c}\mathrm{Al}(\mathrm{OH})_{3}(100 \%) \\
\mathrm{ZnO}(54 \%)\end{array}$ & $\begin{array}{c}\mathrm{ZnO}(100 \%) \\
\mathrm{Al}(\mathrm{OH})_{3}(75 \%)\end{array}$ & $\begin{array}{c}\mathrm{ZnAl}_{2} \mathrm{O}_{4}(100 \%) \\
\mathrm{ZnO}(50 \%)\end{array}$ \\
\hline $\mathrm{Zn}\left(\mathrm{NO}_{3}\right)_{2}+\mathrm{Al}\left(\mathrm{NO}_{3}\right)_{3}$ & $\begin{array}{c}\mathrm{ZnAl}_{2} \mathrm{O}_{4}(100 \%) \\
\text { много РАФ }\end{array}$ & $\begin{array}{c}\mathrm{ZnAl}_{2} \mathrm{O}_{4}(100 \%), \gamma-\mathrm{Al}_{2} \mathrm{O}_{3} \\
(46 \%), \text { много РАФ }\end{array}$ & $\begin{array}{l}\mathrm{ZnAl}_{2} \mathrm{O}_{4}(100 \%) \\
\mathrm{ZnO}(29 \%)\end{array}$ \\
\hline
\end{tabular}

Примечание. РАФ - рентгеноаморфная фаза.

много РАФ. В процессе МВО не удается получить продукт, содержащий преимущественно цинковую шпинель. В дальнейшем подготовленные смеси реагентов подвергали термическому обжигу $\left(1000{ }^{\circ} \mathrm{C}, 1\right.$ ч).

Данные РФА продуктов после двойной термообработки представлены на рис. 6.

Ганит образуется при составлении реакционных смесей из всех пар прекурсоров. 100\%-ные пики всегда соответствуют шпинели, за исключением смеси, состоящей из двух гидроксидов. Продукт обжига сравнительно хорошо закристаллизован. Наибольшее содержание продукта характерно для смеси нитратов $\mathrm{Zn}$ и $\mathrm{Al}$, а наименьшее (менее 50 \%) - для смеси гидроксидов. Для повышения выхода следует повысить длительность микроволновой и/или термической обработки или же поднять температуру заключительного обжига. Возможно также использование механоактивации.

Было проверено действие истирания в шаро-кольцевой мельнице и ударно-истирающее воздействие в планетарной мельнице (табл. 3). Любой из видов предварительной обработки повышает выход продукта.

Истирающая обработка в шаро-кольцевой мельнице малоэффективна. Наиболее результативна предварительная МО в планетарной мельнице, обеспечивающая почти 100\%-ные содержание шпинели после обжига (рис. 7).

ИК(FTIR)-спектр ганита из смеси гидроксидов Zn и $\mathrm{Al}$ после обработки в планетарной мельнице и обжига содержит очень слабые полосы адсорбированной воды ( 3430 и $1625 \mathrm{~cm}^{-1}$ для валентных и деформационных колебаний), а также полосы в области 500-830 см${ }^{-1}$, связанные с колебаниями Zn-O, Al-O и Zn-O-Al (рис. 8).

C повышением температуры обжига дефекты решетки частично залечиваются; вследствие рекристаллизации растет размер областей когерентного рассеяния и уменьшается удельная поверхность порошка (табл. 4). Упорядочение структуры приводит к уплотнению и уменьшению параметра ячейки, приближаясь к теоретической величине (0.7910 нм [2]). 


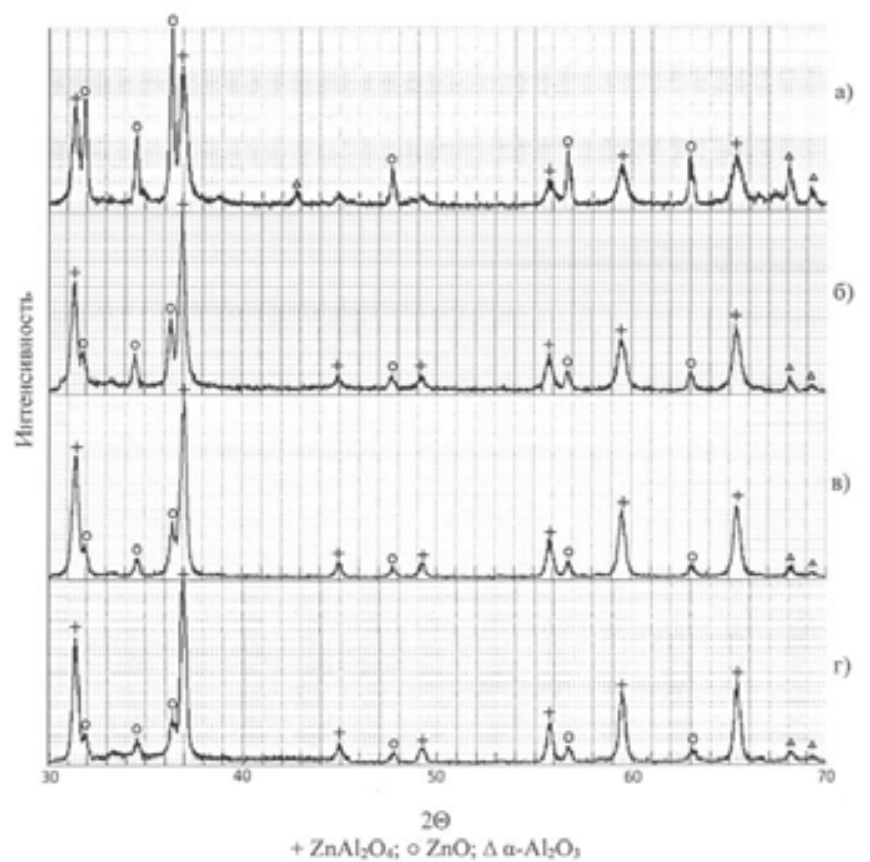

Рис. 6. Дифрактограммы реакционных смесей, подвергнутых микроволновой обработке (15 мин) после обжига в термической печи $\left(1000{ }^{\circ} \mathrm{C}, 1\right.$ ч): $a-\mathrm{Zn}(\mathrm{OH})_{2}$ и $\mathrm{Al}(\mathrm{OH})_{3} ; \sigma-\mathrm{Zn}\left(\mathrm{NO}_{3}\right)_{2}$ и $\mathrm{Al}(\mathrm{OH})_{3} ;$ в $-\mathrm{Zn}(\mathrm{OH})_{2}$ и $\mathrm{Al}\left(\mathrm{NO}_{3}\right)_{3} ; 2-\mathrm{Zn}\left(\mathrm{NO}_{3}\right)_{2}$ и $\mathrm{Al}\left(\mathrm{NO}_{3}\right)_{3}$

Fig. 6. Diffractograms of reaction mixtures after microwave treatment for 15 minutes and subsequent burning in a thermal kiln at $1000^{\circ} \mathrm{C}$ for 1 hour: $a-\mathrm{Zn}(\mathrm{OH})_{2}$ and $\mathrm{Al}(\mathrm{OH})_{3} ; \sigma-\mathrm{Zn}\left(\mathrm{NO}_{3}\right)_{2}$ and $\mathrm{Al}(\mathrm{OH})_{3} ; \beta-\mathrm{Zn}(\mathrm{OH})_{2}$ and $\mathrm{Al}\left(\mathrm{NO}_{3}\right)_{3} ; 2-\mathrm{Zn}\left(\mathrm{NO}_{3}\right)_{2}$ and $\mathrm{Al}\left(\mathrm{NO}_{3}\right)_{3}$

Таблица 3. Выход $\mathrm{ZnAl}_{2} \mathrm{O}_{4}$ из смесей, подвергнутых микроволновой и механической обработке, после обжига $\left(1000^{\circ} \mathrm{C}, 1\right.$ ч)

Table 3. Conversion of $\mathrm{ZnAl}_{2} \mathrm{O}_{4}$ from mixtures after microwave treatment, mechanical activation and subsequent kilning at $1000{ }^{\circ} \mathrm{C}$ for one hour

\begin{tabular}{|c|c|c|c|c|}
\hline \multirow{2}{*}{$\begin{array}{c}\text { Состав } \\
\text { реакционной смеси }\end{array}$} & $\begin{array}{c}\text { Выход } \\
\text { шпинели, \%, без } \\
\text { предварительной } \\
\text { обработки }\end{array}$ & \multicolumn{3}{|c|}{$\begin{array}{c}\text { Выход шпинели, \%, } \\
\text { после предварительной обработки }\end{array}$} \\
\cline { 3 - 5 } & $31 \pm 8$ & $44 \pm 8$ & $\begin{array}{c}\text { ш шаро-кольцевой } \\
\text { мельнице }\end{array}$ & $\begin{array}{c}\text { в планетарной } \\
\text { мельнице }\end{array}$ \\
\hline $\mathrm{Zn}(\mathrm{OH})_{2}+1(\mathrm{OH})_{3}$ & $49 \pm 9$ & $78 \pm 15$ & $54 \pm 14$ & $94 \pm 17$ \\
\hline $\mathrm{Zn}(\mathrm{OH})_{2}+\mathrm{Al}\left(\mathrm{NO}_{3}\right)_{3}$ & $48 \pm 8$ & $71 \pm 9$ & $56 \pm 9$ & $98 \pm 16$ \\
\hline $\mathrm{Zn}\left(\mathrm{NO}_{3}\right)_{2}+\mathrm{Al}\left(\mathrm{OH}_{3}\right.$ & $61 \pm 9$ & $84 \pm 16$ & $53 \pm 8$ & $95 \pm 9$ \\
\hline $\mathrm{Zn}\left(\mathrm{NO}_{3}\right)_{2}+\mathrm{Al}\left(\mathrm{NO}_{3}\right)_{3}$ & & & & $74 \pm 18$ \\
\hline
\end{tabular}




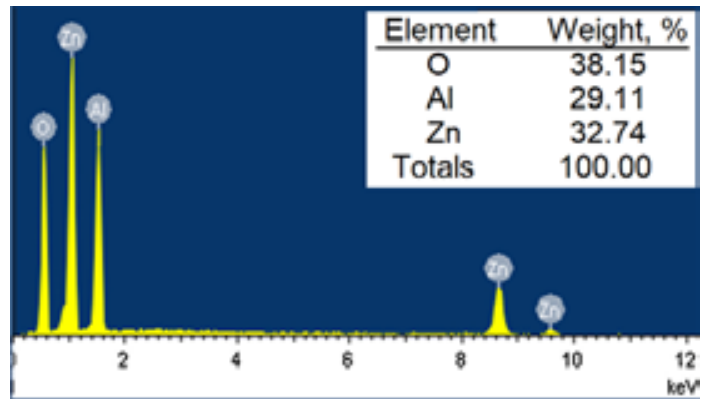

Рис. 7. Энергодисперсионный спектр продукта обжига реакционной смеси из $\mathrm{Zn}\left(\mathrm{NO}_{3}\right)_{2}$ и $\mathrm{Al}\left(\mathrm{NO}_{3}\right)_{3}$ после обработки в планетарной мельнице

Fig. 7. Energy-despersive spectrum of $\mathrm{Zn}\left(\mathrm{NO}_{3}\right)_{2}$ and $\mathrm{Al}\left(\mathrm{NO}_{3}\right)_{3}$ reaction mixture kilning products after treatment in a planetary mill

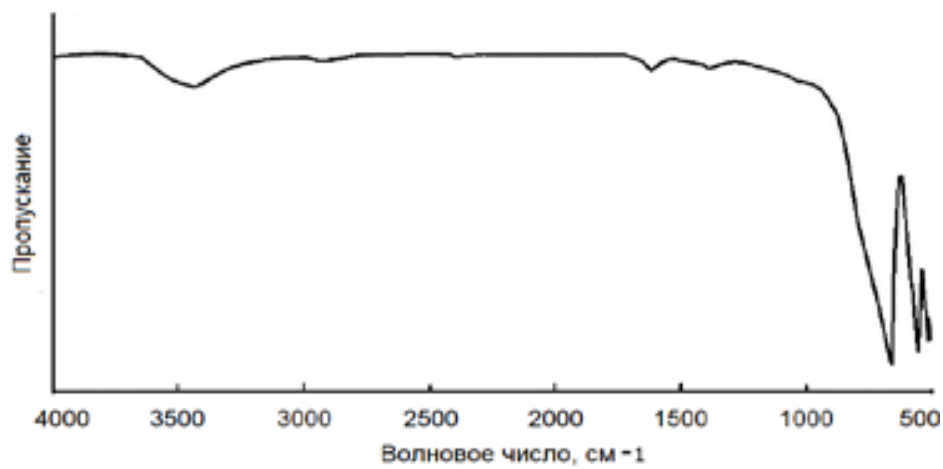

Рис. 8. FTIR-спектр продукта обжига реакционной смеси из $\mathrm{Zn}(\mathrm{OH})_{2}$ и $\mathrm{Al}(\mathrm{OH})_{3}$ после обработки в планетарной мельнице

Fig. 8. FTIR-spectrum of $\mathrm{Zn}(\mathrm{OH})_{2}$ and $\mathrm{Al}(\mathrm{OH})_{3}$ reaction mixture kilning products after treatment in a planetary mill

Таблица 4. Структурные характеристики ганита из обожженной смеси нитратов Zn и Al после обработки в планетарной мельнице

Table 4. Structural characteristics of gahnite made from kilned mixture of $\mathrm{Zn}$ and Al nitrates after treatment in a planetary mill

\begin{tabular}{|c|c|c|c|}
\hline $\begin{array}{c}\text { Температура } \\
\text { обжига, }{ }^{\circ} \mathrm{C}\end{array}$ & $\begin{array}{c}\text { Размер областей когерентного } \\
\text { рассеяния, нм }\end{array}$ & $\begin{array}{c}\text { Параметр ячейки } \\
\mathrm{a}, \mathrm{нм}\end{array}$ & $\begin{array}{c}\text { Удельная поверхность, } \\
\mathrm{M}^{2} / \Gamma\end{array}$ \\
\hline 800 & 12.3 & $0.8101 \pm 0.0004$ & $80.3 \pm 0.6$ \\
\hline 1000 & 18.5 & $0.8094 \pm 0.0004$ & $71.5 \pm 0.4$ \\
\hline 1200 & 28.6 & $0.8083 \pm 0.0003$ & $28.1 \pm 0.5$ \\
\hline 1400 & 44.1 & $0.8060 \pm 0.0002$ & $6.0 \pm 0.2$ \\
\hline
\end{tabular}




\section{Заключение}

Исследовано влияние различных способов подготовки реакционных смесей (механическая, микроволновая обработка) из гидроксидных и солевых прекурсоров на синтез цинковой шпинели. Оценены константы скорости реакции образования $\mathrm{ZnAl}_{2} \mathrm{O}_{4}$. Максимальный выход продукта при $1000{ }^{\circ} \mathrm{C}$ наблюдается при использовании нитратов цинка и алюминия. Предварительная механическая обработка в планетарной мельнице позволяет получить практически монофазный продукт после обжига при 1000 드. Близкий результат можно получить при предварительной СВЧ-обработке смеси нитратов Zn и Al.

\section{Список литературы / References}

1. Ефимов А.И., Белорукова Л.П., Василькова И.В. Свойства неорганических соединений: Справочник. Л.: Химия, 1983. 392 с. [Efimov A.I., Belorukova L.P., Vasil 'kova I.V. Inorganic compounds properties: Reference guide. Leningrad: Chemistry, 1983. 392 p. (In Russ.)].

2. Sampath S.K., Kanhere D.G., Pandey R. Electronic structure of spinel oxides: zinc aluminate and zinc gallate. J. Phys.: Condens. Matter. 1999. Vol. 11, P. 3635-3644.

3. Van der Laag N.J., Snel M.D., Magusin P.C.M.M., de With G. Structural, elastic, thermophysical and dielectric properties of zinc aluminate $\left(\mathrm{ZnAl}_{2} \mathrm{O}_{4}\right)$. J. Eur. Cer. Soc. 2004. Vol. 24 (8), P. 24172424.

4. Yong X., Ping F., Baohua Zh. Optical properties of transparent $\mathrm{ZnAl}_{2} \mathrm{O}_{4}$ ceramics: A new transparent material prepared by spark plasma sintering. Mater. Letters. 2014. Vol. 123, P. 142-144.

5. Yang M., Li Sh., Chen G. High-temperature steam reforming of methanol over $\mathrm{ZnO}-\mathrm{Al}_{2} \mathrm{O}_{3}$ catalysts. Appl. Catal. B: Environ. 2011. Vol. 101 (3-4), P. 409-416.

6. Sumathi S., Kavipriya A. Structural, optical and photocatalytic activity of cerium doped zinc aluminate. Solid State Sciences. 2017. Vol. 65, P. 52-60.

7. Прокофьев В.Ю., Кульпина Ю.Н., Гордина Н.Е. Приготовление алюмоцинкового сорбента с использованием ультразвуковых воздействий. Изв. вузов. Сер. Химия и хим. технол. 2015. T. 58 (11), C. 50-53. [Prokof'ev V.YU., Kul'pina YU.N., Gordina N.E. Manufacturing zincalume sorbent agent using ultrasonic treatment. Izv. Vyssh. Uchebn. Zaved. Khim. Khim. Tekhnol. 2015. Vol. 58 (11), P. 50-53. (In Russ.)]

8. Fernández-Osorio A., Rivera C.E., Vázquez-Olmos A. Luminescent ceramic nano-pigments based on terbium-doped zinc aluminate: Synthesis, properties and performance. Dyes and Pigments. 2015. Vol. 119, P. 22-29.

9. Mekprasart W., Worasawat S., Tangcharoen T. Characterization and effect of calcination temperature on structural properties of spinel zinc aluminate synthesized via co-precipitation process. Phys. Status Solidi C. 2015. Vol. 12 (6), P. 624-627.

10. Guo X., Yin P., Lei W. [et al.] Synthesis and characterization of monolithic $\mathrm{ZnAl}_{2} \mathrm{O}_{4}$ spinel with well-defined hierarchical pore structures via a sol-gel route. J. Alloys and Compounds. 2017. Vol. 727, P. 763-770.

11. Ianoş R., Băbuţă R., Păcurariu C. Combustion synthesis of $\mathrm{ZnAl}_{2} \mathrm{O}_{4}$ powders with tuned surface area. Ceram. Intern. 2017. Vol. 43 (12), P. 8975-8981.

12. Dwibedi D., Murugesan C., Leskes M. Role of annealing temperature on cation ordering in hydrothermally prepared zinc aluminate $\left(\mathrm{ZnAl}_{2} \mathrm{O}_{4}\right)$ spinel. Mater. Res. Bull. 2018. Vol. 98, P. 219-224. 
13. Peillon N., Zu F., Meunier C. In-situ studies on preparation of $\mathrm{ZnAl}_{2} \mathrm{O}_{4}$ spinel using microwave reactive sintering technique. Mater. Lett. 2016. Vol. 167, P. 77-80.

14. Fabián M., Bottke P., Girman V. A simple and straightforward mechanochemical synthesis of the far-from-equilibrium zinc aluminate, $\mathrm{ZnAl}_{2} \mathrm{O}_{4}$, and its response to thermal treatment. $R S C A d v$. 2015. Vol. 5, P. 54321-54328. 